\title{
Research of Compensate Fuzzy Nerve Net Control on Stainless Steel Grind Coefficient of Assembly Technology
}

\author{
Zhanjun LIU \\ Faculty of Aerospace Engineering \\ Shenyang Aerospace University, Shenyang \\ Shenyang, China \\ e-mail: liuzhanjun3333@163.com
}

\begin{abstract}
It is proposed the evaluation theory of inverse analysis arithmetic, and it is introduced the evaluated equation on compensate fuzzy control by stainless steel, the affecting gene model of stainless steel on compensate fuzzy control is built. The application of installation process is introduced. The goal specimen is combined with the special predict results of affecting gene. The result is proved that subdued stress of inverse analysis attained is treated as true subdued stress, it is obtained the accuracy prediction of grind coefficient of ring constringent test on inverse analysis.
\end{abstract}

Keywords-assembly technology; compensate fuzzy nerve net control; stainless steel; grind coefficient

\section{INTRODUCTION}

The numerical simulation of metal forming technology requires the material performance of precise information, and the precision of the simulation to a large extent depends on the correctness of which the mechanical and thermal boundary conditions are described. Simulation of thermal mechanical process is one of the challenges of compression, tension and torsion of these various plastic deformation experiment, whose parameters is determination. The advantages and disadvantages of these experiments is clear for the interpretation of the results is clear. The main difficulty is that the strain of uneven, local strain, friction and the influence of heat due to deformation and friction. Therefore, the purpose of the study is to find a way to eliminate the adverse effect during plastic deformation, and the rheological parameters have nothing to do with these phenomena and friction parameters. Because when the rheological parameters are decided, friction coefficient must be assumed, and vice versa, and the task of the rheological parameters and is still complicated with friction parameters, to solve the task becomes more important. Although the friction plays a main role in the process of metal deformation, but it is hard to accurately determine the friction equation and friction in the role of material rheological parameter. In this paper, it studies at the same time in a kind of plastic measurement to determine the rheological parameters and friction of complicated problems, which is using tertiary inverse algorithm to calculate and study the problem. Inverse problem of the metal deformation process is ignored and individual cases, which is using tertiary inverse algorithm to compute three inverse algorithm, it basically has the following three aspects[1,2].
Compensation fuzzy neural network is a combination of fuzzy logic and neural network hybrid system, it is consisted of fuzzy neurons and decision-making for controlling, the fuzzy neuron are defined as fuzzy arithmetic and fuzzy reasoning, fuzzy arithmetic compensation and the blur operation. Networks can train from the initial definition of right or error definition of fuzzy rules, making the network higher fault tolerance, the system more stabling. In compensation fuzzy neural networks, it is used in fuzzy operation dynamic and global optimization algorithm, and in the learning algorithm of neural network, fuzzy and dynamic optimization of the compensation arithmetic is made in the network of more adaptive and optimization. Network can not only adjust the input and output fuzzy membership function, but also can be compensated by means of dynamic optimization of adaptive fuzzy inference logic algorithm, the network parameters have clear physical meaning $[3,4]$.

\section{THE INTERNAL VARIABLE MODEL PARAMETERS AND FRICTION PROPERTIES}

\section{A. Decided Internal Variable Model Parameters}

The calculation results are using the inverse analysis, that is three inverse analysis. using the formula to define the objective function can estimate stamping contact friction on the surface of the sample. Two rheological model parameters are using two step inverse algorithm to calculate, friction parameters and rheological parameter are at the same time.The objective function is defined as type[5,6].

\section{B. Calculating Friction Factor}

Each test experiment firstly determines the unknown mc alone. Friction Facto equation as follows:

$$
\tau=m_{c} \sigma_{p}\left[\frac{2}{\pi} \tan ^{-1}\left(\frac{v_{s}}{a}\right)\right]
$$

Results show that the compression process of friction coefficient is related to temperature and pressure. Fig.1 shows that compression in the process of prediction sample shape and shape in both cases, the actual sample can determine the friction coefficient method. Known from the figure, they identify that the friction coefficient is very close, and the friction coefficient increases with the rise of temperature and friction factor, with calibration curve, it can get the coulomb friction coefficient about 0.15 , Chen and Kobayashi friction factor is $0.25 \mathrm{MC}$ material, using inverse 
analysis of the friction factor values below Chen and Kobayashi friction factor is valuable. Because the latter approach is based on measuring the coefficient of friction resulting from the ring shape, it is more reliable. Results of Fig. 1 and Fig.2 are confirmed by inverse analysis technology in predicting the accuracy of the coefficient of friction ring compression tests.

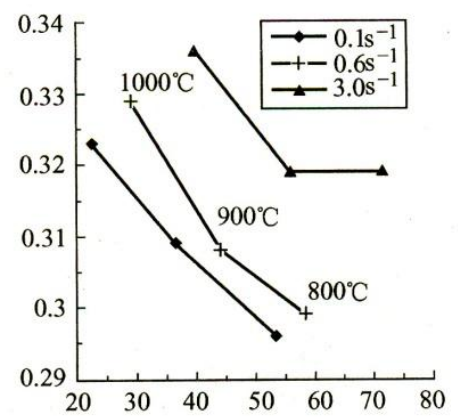

Horizontal axis is $\mathrm{mm}$, vertical axis is dimensionless

Figure 1. Metrical grand gene of different distortion.
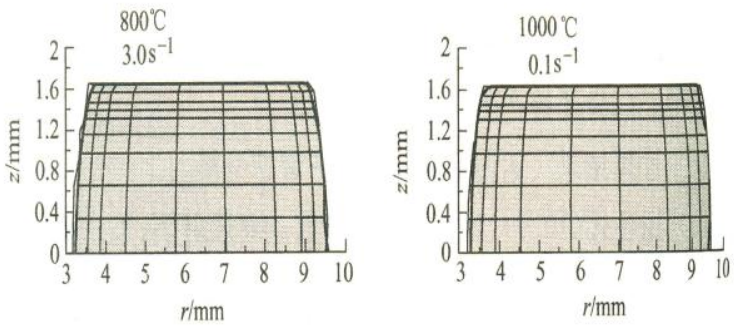

Figure 2. Stainless steel ring shape of prediction and test $1 / 4$ distortion.

\section{ThE Plastic Simulator Is USED TO DETERMINE THE STAINLESS STEEL FRICTION FACTOR}

\section{A. Materials and Experiment}

Experimental materials is for $340 \mathrm{~L}$ of stainless steel, using the axisymmetric compression method, and it uses Gleeble 3800 thermal simulator, deformation dilatometer DIL1 and DIL2 was simulated.Test conditions and sample sizes are shown in Table 1, $\mathrm{T}$ is a standard test temperature, vs is speed for the tool, epsilon is as the strain rate, h0 is high of original sample, the original diameter is d0 samples, hf is the samples of high end, df is sample final diameter. Barrel sample volume is obtained by using the formula.

TABLE I. AXILE SYMMETRY CONSTRINGENT WAY

\begin{tabular}{|c|c|c|c|c|c|c|}
\hline machine & $\mathrm{T} /{ }^{\circ} \mathrm{C}$ & $\mathrm{vs}$ & $\mathrm{h}_{0}$ & $\mathrm{~d}_{0}$ & $\mathrm{~h}_{\mathrm{f}}$ & $\mathrm{d}_{\mathrm{f}}$ \\
\hline GLI & 900 & 0.4 & 16 & 13 & 9 & 15.5 \\
\hline DIL1 & 900 & 0.4 & 11 & 6.0 & 6.1 & 6.75 \\
\hline DIL2 & 900 & 0.4 & 10 & 6.0 & 5.5 & 7.36 \\
\hline
\end{tabular}

\section{B. To Estimate the Coefficient of Friction}

Inverse analysis rely on precision tools and sample contact region friction condition, the deformation after mc sample weight decided the friction factor, the sample weight formula:

$$
\mathrm{V}=(\mathrm{v} 0-\mathrm{vc}) / \mathrm{v} 0 * 100 \%
$$

\section{Established the Compensation Fuzzy Control System of Stainless Steel Friction Factor}

The fuzzy optimization model of metal plates drawing force factor influencing is the key problem of system development [2], and put all the sheet metal deep influence factor affecting factors of power for $U=\left\{\mathrm{u}_{1}, \mathrm{u}_{2}, \ldots, \mathrm{u}_{\mathrm{n}}\right\}$. Satisfy $\mathrm{U}=\mathrm{Ui} \cup \mathrm{U} \mathrm{j}$, and when I indicates whe $\mathrm{n} \mathrm{i} \neq \mathrm{j}, \mathrm{U} \mathrm{i} \cap \mathrm{U} \mathrm{j}=\{0\}$. The among vector of sheet metal deep influence factor affecting factors for $\mathrm{U}=\left\{\mathrm{u}_{\mathrm{i} 1}, \mathrm{u}_{\mathrm{i} 2}, \ldots, \mathrm{u}_{\mathrm{ik}}\right\}$. By introducing the weighted coefficient sets $A=\left(a_{1}, a_{2}, \ldots, a_{n}\right), a_{i}$ present level factors $u \mathrm{i}$, that is weight size of $u \mathrm{i}$. All of the collection of various factors for $V=\left\{v_{1}, v_{2}, \ldots, v_{m}\right\}$ level set.

Based on the ideas above, for $r_{i j}\left(u_{i}, v_{j}\right)$, it is introduced into dynamic variable $X$ to improve, to build the fuzzy membership function of dynamic evaluation formula:of sheet metal drawing force factor influencing, when parameters $\mathrm{x}$ impact conditions, no is composed by parameter condition of the influence $\mathrm{x}$.

Type of dynamic variables, $x$ is used to determine the different period of membership matrix. It is obtained the force of the sheet metal deep impact factor of the basic model of fuzzy dynamic evaluation[7, 8].

$$
\mathrm{B}=\mathrm{A} \circ \mathrm{R}=\mathrm{b} 1 / \mathrm{v} 1+\mathrm{b} 2 / \mathrm{v} 2+\mathrm{bn} / \mathrm{vn}
$$

"A $\circ \mathrm{R}$ " is said "generalized fuzzy synthetic operation," To assess for $v_{j}$ grade of fuzzy subsets of membership, the fuzzy $\mathrm{B}$ vector normalization, according to the maximum membership degree, the result of dynamic testing can be got.

In this paper, the design of compensation fuzzy network evaluation of the sheet metal deep force impact factor, to choice of aluminum alloy plates deep deformation performance parameters as input node, with deep force factor influencing the plank as output node k. 6 input nodes is: the height of deep plank blank, holder force, friction coefficient, hardening exponent, intensity coefficients and thick anisotropic coefficient values.

\section{The Evaluation Structure of Stainless Steel Friction Factor of Compensation Fuzzy Neural Networks}

Compensation fuzzy neural network structure has five layers: input layer, the blurred layer, compensation arithmetic, the blurred layer and output layer. It is based on fuzzy logic system between layer and layer of language variable and fuzzy if-then rules, the worst - the best operation, fuzzy reasoning method and the fuzzy functions. Among them, the each node of first layer is connected to the input vector directly; The each node of second layer represents a linguistic variable values, its role is to calculate 
the input vector belonging to each linguistic variable values of the fuzzy setting membership function; The each node of third layer represents a fuzzy rules, its role is to match the fuzzy rules, and calculates the suitable degree of each rule.

Fuzzy membership function for

$$
\begin{aligned}
& \left.\mu_{A i}^{k}(x)=\exp \mid-\left(\frac{x_{i}-a_{i}{ }^{k}}{\sigma_{i}{ }^{k}}\right)^{2}\right\rceil \\
& \mu_{B_{i}{ }^{k}}(y)=\exp \left\lfloor-\left(\frac{y-b^{k}}{\delta^{k}}\right)^{2}\right\rceil
\end{aligned}
$$

The alpha with sigma is for the center and width of inputting membership functions; B with the delta is as the center and width of the output membership function.

Define input $X=\left(x_{1}, \ldots, x_{n}\right)$, the theory of domain for: $U$ $=\mathrm{U}_{1} \times \mathrm{U}_{2} \ldots \mathrm{U}_{\mathrm{n}}, \mathrm{A}^{\prime}$ is an input fuzzy subset of theory domain in $\mathrm{U}$, according to the fuzzy rules of $\mathrm{k}, \mathrm{B}$ ' is produced in the output field of $\mathrm{V}$ fuzzy subset, the fuzzy reasoning is used in the largest algebra product of synthesis arithmetic[9].

As a result, the system is made up of single valued fuzzy generator, gaussian membership function, the product of reasoning principle, negative - positive compensation algorithm and improved center of the compensation fuzzy neural networks.

In the design of sheet metal deep drawing force impact factor of compensation fuzzy network evaluation in [8], the input node is selected of aluminum alloy sheet deep drawing deformation performance parameters, the output node is the $\mathrm{K}$ value of sheet deep drawing force impact factor. Six specific input nodes as follows: the height of the sheet metal deep drawing, blank holder force, friction coefficient, hardening index, intensity index and thick anisotropic coefficient values.

In assembly technology, the aircraft assembly is the parts, components in accordance with the design and technical requirements of the combination, the formation of a high level of assembly or machine process. Aircraft assembly due to the size of the product, the shape of complex, parts and the number of connections, the amount of labor accounted for about half of the total amount of aircraft manufacturing labor and more, aircraft assembly technology and organization and management, although the local use of more advanced technology, such as the use of laser tracker or computer-assisted theodolite technology to install the type of frame, a small number of automatic drilling riveting technology, simplifying the assembly frame structure. But compared with the developed countries there is still a large gap so the aircraft assembly technology has become a bottleneck restricting the capacity of aircraft manufacturing technology, the development of aircraft digital assembly technology is imminent.

\section{AIRCRAFT DigITAL ASSEMBLY TECHNOLOGY}

Aircraft digital assembly technology system involves aircraft design, parts manufacturing, digital automatic drilling and riveting system, digital interchange coordination, digital advanced measurement and detection and computer software and many other advanced technology and equipment, machinery, electronics, control, computer and other disciplines Cross-integration of high-technology. Its architecture, including aircraft digital assembly of key technologies and digital assembly process equipment, two parts. Aircraft digital assembly of key technologies include: aircraft digital assembly basic technology, application technology and standard specifications Aircraft; digital assembly process equipment mainly include: component digital assembly system, parts of the digital assembly system and aircraft assembly digital assembly production line.

To shorten the product cycle, in the aircraft structure design at the same time should begin tooling design, that is, aircraft products and aircraft tooling parallel design. The design of the tooling is based on the aircraft product data, in the final product data has not yet been determined in the case of tooling design, tooling part of the structure must be independent of the product data. One of the basic ideas for tooling and product parallel design is to change the traditional tooling structure, divide it into two parts: a standard structure that is independent of the product data or requires only basic data and a dedicated structural part that relies on the final product data. The standard part of the assembly frame is mainly column, base, auxiliary support, etc., the special part of the main bar for the positioning of the scale plate, connector positioning, and so on. Special parts are generally smaller in size, design, processing and manufacturing cycle is very short, and no special large-scale processing equipment. Standard structure size, complex structure, often need special large-scale processing equipment, its design, manufacturing cycle is long. The design of the standard structure does not require the final product data or only some basic data, so the aircraft product design can be designed and manufactured at the beginning, when the final version of the product after the release of a short period of time to complete the design and manufacture of special structure. Standard parts and special parts to determine the assembly design method, the assembly is very convenient, and no special installation tools, assembly cycle is short. In this way, within a very short time after the completion of the product design can be put into the product assembly.

Digital assembly coordination method can also be called digital standard tooling coordination method, is an advanced digital standard tooling based on the definition of interchangeable technology, It will ensure that the production process equipment, between the production process equipment and products, product components and components between the size and shape of the coordinated exchange. Digital assembly coordination and tolerance 
allocation technology is to ensure the accuracy of aircraft assembly, and the key to improve the quality of aircraft products. Digital assembly coordination and tolerance allocation technology in the process planning stage to aircraft digital design data based on the digital environment to complete the aircraft coordination program and tolerance program planning to ensure the feasibility of assembly, assembly accuracy and assembly quality.

\section{CONCLUSION}

It is proposed the evaluation theory of inverse analysis arithmetic, and it is introduced the evaluated equation on compensate fuzzy control by stainless steel, the affecting gene model of stainless steel on compensate fuzzy control is built. The application of installation process is introduced. The goal specimen is combined with the special predict results of affecting gene. The result is proved that subdued stress of inverse analysis attained is treated as true subdued stress, it is obtained the accuracy prediction of grind coefficient of ring constringent test on inverse analysis.

\section{REFERENCES}

[1] Boyer, Inverse Analysis For Identification of Parameters During Thermo-Mechanical Tests. Proceedings of Numiform (2001),p281.

[2] Gelin,J.C,Ghouati,O.An Inverse Method for Determining Viscoplastic Properties of Aluminium Alloys,J.Mat.Proc.Techn.(1994), p435 .

[3] Lenard ,J.G.Tribology in Metal Rolling ,Ann.CIRP(2000),p41 .

[4] Schey,J.A.Introduction to Manufacturing Processes (1987), p50-57.

[5] LU Yi,WU Xiao-chun. Research progress of prehardened plastic mould steel. Heat treatment of metals, 2007.

[6] P Kwon, M J Chung and B Pentland. A Grammar based Framework for Integrating Design and Manufacturing. Transactions of the ASME , Journal of Manufacturing Science and Engineering, 2002(4) : p124 .

[7] He Ping.The Research of Tube Forming Parameter Recognition of Compensate Fuzzy Nerve Net Control[C],Advances in Materials, Machinery, Electrical Engineering, 2017.

[8] Liu Zhan jun. Research on Cause of Boron Steel Sheet Size Precision Based on Compensate Fuzzy Nerve Net Control[C], Joint International Information Technology, Mechanical and Electronic Engineering Conference, 2016.

[9] Ming-Xu L I, Zhou Y, Zhao H M, et al. Application of Compensation Fuzzy Neural Networks in Grinding Control[J]. Computer Simulation, 2015. 